\title{
Geometrical Characterization of Wire-and-Arc Additive Manufactured Steel Elements
}

\author{
Vittoria Laghi ${ }^{1, \star}$, Michele Palermo, Giada Gasparini' ${ }^{1}$, Valentina Alena Girelli, \\ Tomaso Trombetti ${ }^{1}$ \\ ${ }^{1}$ Department of Civil, Chemical, Environmental and Materials Engineering, University of Bologna, 40136, Bologna, \\ Italy
}

*Corresponding author: E-mail: vittoria.laghi2@unibo.it; Tel: (+39) 0512093235

Received: 20 March 2019, Revised: 08 May 2019 and Accepted: 14 May 2019

DOI: $10.5185 /$ amlett.2019.0019

www.vbripress.com/aml

\begin{abstract}
In the last decades Additive Manufacturing has gained fundamental importance in the development of digital fabrication for the automotive, aerospace, biomedical and only lately civil engineering field. In particular, the technology of Wireand-Arc Additive Manufacturing, based on a welding process adopted on a robotic arm, is the most suitable to realize structural elements which usually requires large dimensions of the printed outcome, with still a good mechanical response of the printed metal material. The authors have been part of a pioneering work which provides the first insight into the material and geometrical properties relevant to characterize 308LSi stainless steel elements to realize the first 3D-printed steel footbridge to be held in Amsterdam by 2020 and manufactured by the Dutch company MX3D. In detail, the work presents the first results of an intense geometrical study to characterize the intrinsic irregularities of the printed outcome, by means of hand measurements and high-precision 3D scan acquisition of different element types. Copyright $\odot$ VBRI Press.
\end{abstract}

Keywords: 3D printing, additive manufacturing, geometrical properties, experimental tests, 3D scan.

\section{Introduction}

Over the last decade, the application of Computer-Aided Design (CAD) has brought to a new design approach resulting in novel forms, by means of three-dimensional computer modeling and digital fabrication methods, further developed in computerized tools for architecture, structure and civil engineering which have gained more and more influence $[\mathbf{1 , 2}]$.

On the other hand, manufacturing process has seen the advent of automation since the beginning of the $21^{\text {st }}$ century in almost all production domains, from automotive to aerospace, from biomedical to mechanical, with the exception of construction field. Indeed, in the building sector the use of automation has always been challenging due to several intrinsic factors, from the large dimensions of the outcomes to the limitation in the material to be adopted in the automated system [3]. Only recently, Additive-Manufacturing (AM) based technologies, already widely adopted in other sectors such as aerospace, automotive and biomedical engineering, are starting to be applied in structural engineering field as well [4-10].

The term Additive Manufacturing refers to the process of depositing layer upon layer of material, as opposed to the subtractive manufacturing, typically adopted, producing a final $3 \mathrm{D}$ object made in plastic, resin, rubber, ceramic, concrete and metal. Concerning the latter material, recent reviews [11-14] present in literature distinguish three main categories of metal AM processes: (i) Powder Bed Fusion (PBF); (ii) Directed Energy Deposition (DED) and (iii) sheet lamination.

Although extensive research effort has been paid on studying the mechanical characteristics of metal PBF processes [15-20], due to its intrinsic limitations in the dimensions of the printed outcomes, the application in structural engineering field is not of relevance. On the other hand, among the DED processes, Wire-and-Arc Additive Manufacturing (WAAM) seems to be the most suitable one for the construction industry, as it consists of a "printing head" at the top of a robotic arm, allowing for the realization of elements without theoretical dimensional constraints. In fact, it consists of an electric arc as heat source and wire as feedstock, by means of offthe-shelf welding equipment, such as welding power source, torches and wire feeding system, while motion is provided by either robotic system or computer numerical-controlled gantries [21-24].

Among the various metal Additive Manufacturing technologies, Wire-and-Arc Additive Manufacturing (WAAM) process has been recently adopted by the Dutch company MX3D [25] to realize the first 3Dprinted steel footbridge which will be placed in the city center of Amsterdam by 2020 [26-27]. 
However, in order to obtain pieces of large dimensions, higher printing velocities are required, resulting in larger geometrical imperfections with respect to the digital model. Therefore, much effort is needed for a proper assessment of both the geometrical and mechanical characterization of the outputs from Wire-and-Arc Additive Manufacturing (WAAM) process. Previous research has been widely focused on characterizing WAAM elements in titanium alloys [28, 29] and very recently on stainless steel [30-32], although mainly focused on the microstructure and metallurgic properties, and not explicitly investigated for structural engineering purposes.

\section{Challenges related to WAAM process}

Concerning WAAM process, a great source of uncertainty is the outcome of the printing process, as a metal material with characteristics in terms of both geometry and mechanical properties which differ from the traditionally fabricated cold-formed metal elements. In fact, first research effort on such innovative manufacturing technology produced results in terms of main material properties (Young's modulus, yielding stress, ultimate tensile strength, ultimate tensile strain) and geometrical accuracy (thickness, cross-sectional area, net shape) not in line with the typical behavior of traditionally manufactured metal materials. In detail, recent research on stainless steel produced values of Young's modulus of the order to $60 \%$ to $100 \%$ reduction with respect to the traditional values proposed for international standard provisions (i.e. European building code EN 1993:1-4 [33]) for traditionally formed stainless steel parts. Furthermore, studies on microstructure of WAAM elements confirmed a certain influence of the material structure and behavior with the adopted printing parameters [30-32]. The fundamental process parameters are (i) the current and its voltage, (ii) the wire diameter, (iii) the wire-feed rate, (iv) the welding speed and (v) the vertical printed layer height. Therefore, it becomes crucial to properly characterize WAAM metal parts related to the specific process parameters, in terms of geometrical accuracy and mechanical response.

For structural engineering applications, the need of high welding velocity for a rapid realization of structural elements of such proportions plays a crucial role for the specific characteristics of the printed parts, as it induces geometric inaccuracy of the outcomes, both in terms of surface roughness and lack of straightness of the elements. For a given element to be printed, a digital model from which the printing head reads the coordinates of the points defining step by step the position of the welded layer is created with Rhinoceros software [34]. However, due to intrinsic inaccuracy of the printing process, each ideal point of the digital model has a real counterpart whose position is not exactly the one of the digital model, as it is affected by an error.

The specifications of the innovative manufacturing process are crucial to derive the response in terms of structural performances of the printed elements. The large velocity of deposition used to realize the specimens (average welding speed of 0.5 to $2 \mathrm{~kg} /$ hour) has induced some considerable geometric irregularities which should be properly taken into account both in the design and construction phases. Moreover, the heating process induces some non-negligible residual stresses and deformation of the crystalized structure of the stainless steel, which should be further analyzed as well. For instance, Fig. 1 provides different views of typical tubular elements manufactured by MX3D [25] using the continuous printing strategy. The figures allow to visually appreciate the typical geometrical imperfections resulting from the manufacturing process: cross-section shape irregularity (Fig. 1a), lack of straightness (Fig. 1b) and surface roughness (Fig. 1c). These geometrical imperfections together with the specific material mechanical behavior related to the printing parameters affect the structural response of the printed elements and have to be addressed in order to provide valuable information to be used in the structural design. (a)

(b)
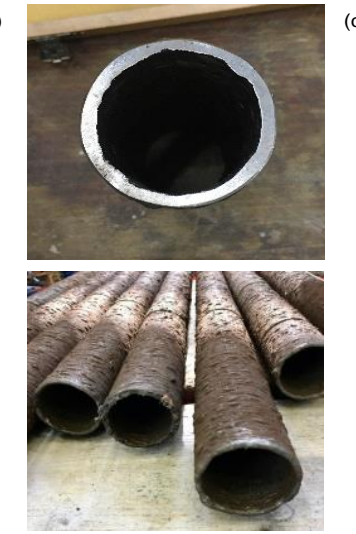

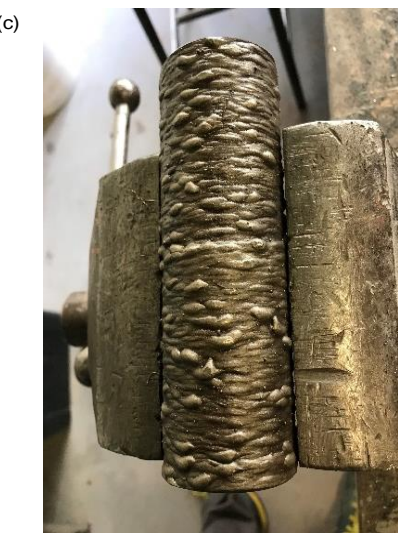

Fig. 1. Geometric issues of WAAM elements: (a) cross-sectional irregularity; (b) lack of straightness; (c) surface roughness.

\section{The experimental campaign}

The work is focused on the WAAM process adopted by MX3D, which makes use of a gas metal arc welding, a process characterized by a continuous wire electrode which is drawn from a reel by an automatic wire feeder. Two different printing strategies have been explored by MX3D: a so-called continuous printing, meaning that the material is deposited in continuous, and a so-called dotby-dot printing, meaning that the material is deposited by successive points. The effects of these strategies on the metallurgic characteristics have been also analyzed in $[30-32]$.

A wide experimental campaign has been carried out starting from January 2017 (and still on-going) at the Topography and Structural Engineering Labs from University of Bologna, in order to assess the geometrical and mechanical properties of real-scaled specimens realized with WAAM process.

In particular, 308LSi austenetic stainless steel has been adopted as wire feed, and the specimens realized according to the following ranges of parameters: $1-\mathrm{mm}$ wire diameter, 0.6 to $2 \mathrm{~mm} / \mathrm{min}$ wire-feed rate, 15 to 
$30 \mathrm{~mm} / \mathrm{sec}$ welding speed, 1 to $3 \mathrm{~mm}$ vertical printed layer height and an average total printing speed of 0.5 to $2 \mathrm{~kg} /$ hour [35]. Such parameters have been calibrated by MX3D in order to realize fully-dense real-scale elements for structural engineering applications.

The phases of the experimental campaign have been defined in order to address the two main issues relevant from a structural design point of view, namely geometrical irregularities and mechanical properties. Further explanation of the first experimental campaign is presented in a more extensive work submitted in a peer reviewed journal [35].

The work here presented is focused on the geometrical characterization of the imperfections due to the printing process, i.e. cross-section irregularities, surface roughness and lack of straightness. Since the amount of imperfections due to the manufacturing process may differ depending on the specific geometry to be printed, the specimens considered are both "dogbone" shaped elements cut from rectangular plates and hollow circular tubes (both stub columns and long tubes). Different types of measurements have been compared including: (i) punctual hand measurements with digital caliper with specific attention on the thickness for the "dog-bone" specimens and both thickness and outer diameter for the tubular specimens; (ii) volume-based measurements (from the Archimedes' principle) to obtain effective values of thickness for both types of specimens; (iii) 3D scan for a detailed evaluation of cross-section irregularities, surface roughness and lack of straightness of both types of elements.

\section{Geometrical characterization of WAAM elements}

As previously mentioned, when it comes to WAAM technology the accuracy in the printed outcome is directly influenced by the high velocities required to realize structural elements of big dimensions in reasonable amount of time. Therefore, it is necessary to characterize the printed material both on the geometric point of view as well as for its mechanical properties.

Considering the digital model, taken as input in the printing process, the geometrical dimensions are nominal values, which however differ with respect to the printed outcome, given the inherent imperfections due to surface roughness of the outcome, lack of straightness and irregularity in the cross-section.

For the current project, two types of geometries have been studied: plates and tubes.

Referring to a generic plate, the digital model has a nominal value of thickness $t_{n}$ constant along the surface of the element. However, the printed outcome results in a value of thickness which is variable and depends on the position of the piece along the plate (taking a coordinate system $\left.\mathrm{x}, \mathrm{y}: t_{\text {real }}=t_{\text {real }}(x, y)\right)$. Therefore, based on the printing direction, the surface roughness can have a different influence on the mechanical behavior of the specimens cut along the printing direction and those cut perpendicular to that. (Fig. 2)
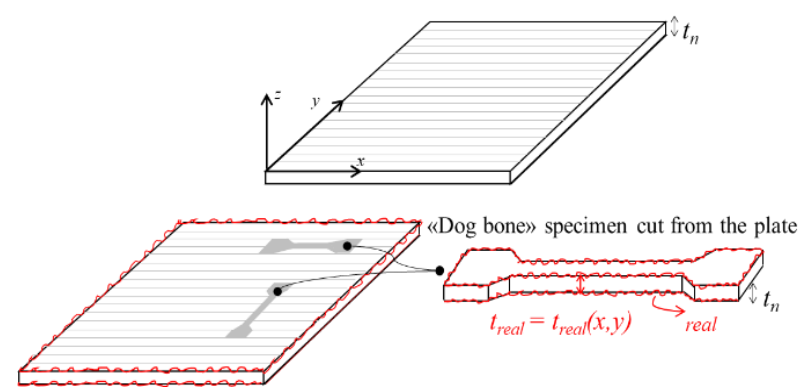

Fig. 2. Digital model vs. printed outcome of a WAAM plate element.

Considering the tubular elements, the input digital model is constituted by a circular-hollow cross-sectional tube, having constant nominal thickness and outer diameter $D_{n}, s_{n}$. Due to the imperfections which are intrinsic in the printing process, the printed outcome is a tubular element with a non-perfectly hollow circular cross-section which varies over the height and a nonstraight longitudinal axis (taking a cylindrical coordinate system z, $\theta: D_{\text {real }}=D_{\text {real }}(\theta, z)$ and $\left.s_{\text {real }}=s_{\text {real }}(\theta, z)\right)$ (Fig. 3).

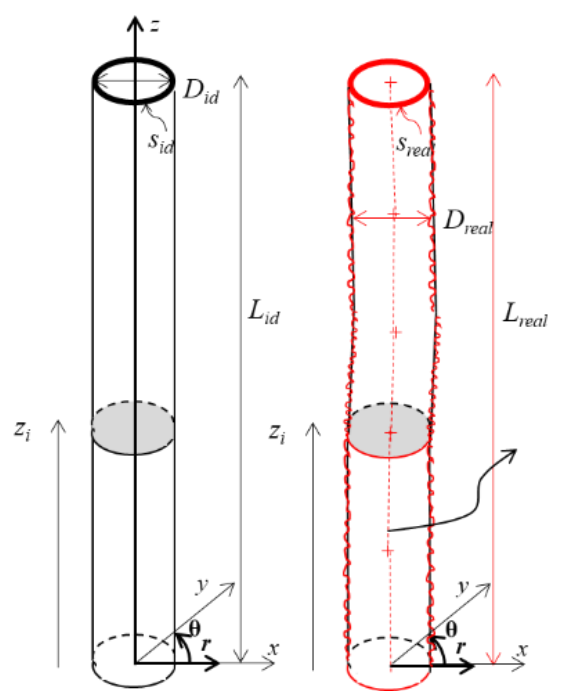

Fig. 3. Digital model vs. printed outcome of a WAAM tubular element.

Therefore, the first phase of the experimental campaign has been the geometrical characterization of the imperfections due to the printing process adopted in the current research, i.e. cross-section irregularities, surface roughness and lack of straightness.

In order to assess the main geometrical parameters of Wire-and-Arc Additive Manufactured elements, the following measuring systems have been used: (i) highprecision caliper (with a nominal value of $0.02 \mathrm{~mm}$ ) for hand measures of thickness of both planar and tubular specimens; (ii) analogic hydraulic scale, to take the volume-based measures of the average thickness for both types of specimens; (iii) 3D scanning acquisition system for the precise evaluation of the thickness variation due to surface roughness for both types of specimens. Fig. 4 shows the equipment adopted for the different types of measures taken. 
(a)

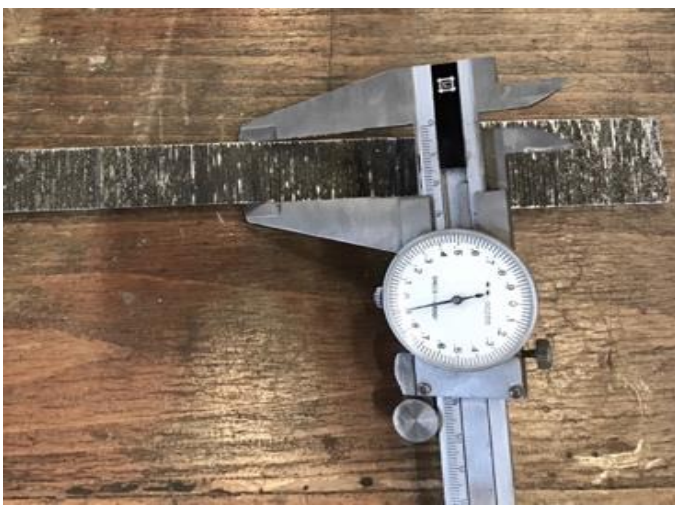

(b)

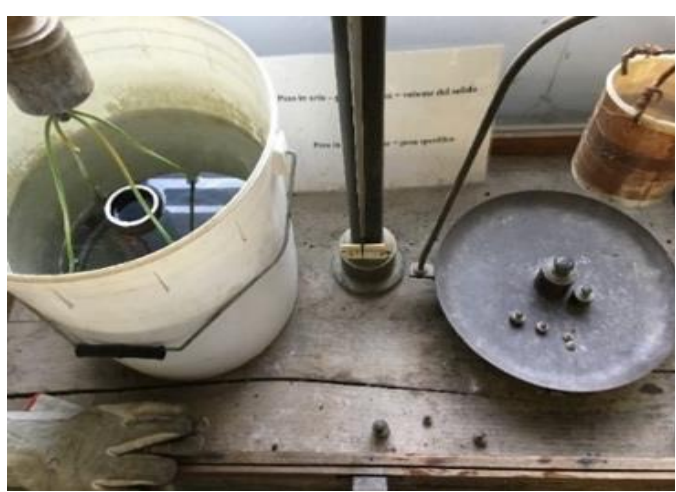

(c)

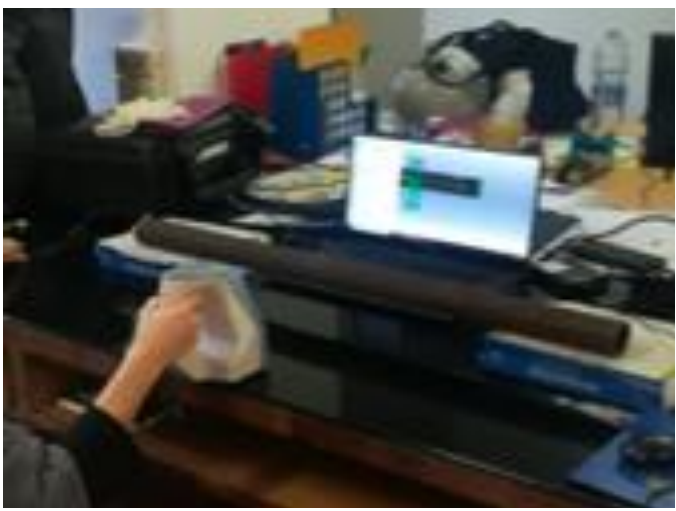

Fig. 4. The measurement set-up for the geometrical characterization of WAAM elements: (a) high precision caliper; (b) hydraulic scale; (c) Artec Spider 3D scanner.

In particular, a limited number of specimens from both types have been first scanned by means of high resolution 3D scanning. It consists of an optical noncontact measuring system based on the blue light, therefore based on the principles of topographic triangulation: the projector projects a fringe pattern of blue light on the object surface and the pattern appears distorted due to the surface shape. In particular, two cameras capture the distortion to calculate the 3D coordinate measurements, acquiring full-field scans of a volumetric area and collecting millions of points per scan.

The details of the structured-light projection Artec Spider [37] scanner used are the following: weight of $0.85 \mathrm{~kg}$, dimensions of $190 \times 140 \times 130 \mathrm{~mm}$, and acquisition speed of $1 \mathrm{mln}$ points/sec for metrology applications. The 3D resolution obtained is of 100 points $/ \mathrm{mm} 2$, with a 3D point accuracy of $0.05 \mathrm{~mm}$ for a medium field size of $90 \times 70 \mathrm{~mm}$ and a working distance of $0.25 \mathrm{~m}$.

Fig. 5 shows details of the obtained 3D model of one of the tubular elements, consisting of around 40 milions of triangular elements, with a mean size of about $0.1 \mathrm{~mm}$. In particular, comparison between the real scanned tube (blue model) and the ideal digital model (grey model) allowed to draw some conclusions concerning the variation of surface roughness along the dimension of the specimen, as well as compute a better estimate of the effective cross-sectional area of the specimen, to be adopted for further mechanical characterization. Details of the work are presented in $[\mathbf{3 5}, \mathbf{3 6}]$.

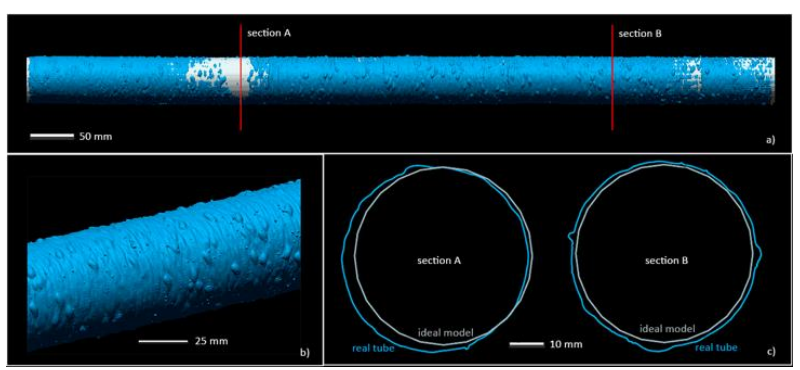

Fig. 5. 3D model of the real printed tube (blue) obtained by 3D scanning, compared with the ideal digital model (grey).

Results of the geometrical characterization of both planar and tubular specimens are here summarized. As far as the planar specimens are concerned, the measurements indicated that, on average, the thickness obtained from caliper measurements is slightly larger $(+13 \%)$ than the nominal value, from the ideal digital model. On the contrary, the value of thickness obtained from volume measurements is slightly less $(-2 \%)$ than the nominal value from the digital model. Therefore, an average ratio between the nominal value and thickness taken from volume measurements of $\chi=1.16$ has been calibrated and used in order to "correct" the crosssectional area to be considered for the mechanical characterization [35].

As far as tubular specimens are concerned, results show that the error in computing the outer diameter is almost negligible for structural engineering purposes. The thickness values obtained from the caliper measures are on average slightly larger than the nominal value $(+5 \%)$, as for the previous case, whereas the value obtained from volume measurements is smaller than the nominal value $(-23.7 \%)$.

Further details are presented in $[\mathbf{3 5}, \mathbf{3 6}]$.

\section{Conclusions}

In the paper, the first results of a wide experimental campaign conducted at University of Bologna labs on Wire-and-Arc Additive Manufacturing (WAAM) stainless steel are presented. In particular, the focus has been on the geometrical characterization of different 
types of WAAM outcomes (plates and tubes) to draw some first conclusions on the surface irregularities typical of this type of process.

In particular, the attention has been paid on two types of specimens, planar and tubular, studied in terms of variation of cross-sectional area. Results obtained from high-precision hand measurements, volume-based measurements and 3D scanning acquisition system have been analyzed and compared. In detail, the effort has been focused in: (i) evaluating the discrepancies between the nominal and the actual (effective) thickness of the specimens extracted from the planar plates which have been quantified, on average, in the order of $+-0.5 \mathrm{~mm}$ (+$10 \%$ of the nominal value); (ii) evaluating the discrepancies between the nominal and the actual (effective) thickness and cross-section outer diameter of hollow tubes, quantified in the order of $-1 \mathrm{~mm}$ for thickness (-20\% of the nominal value) and $-2 \mathrm{~mm}$ for cross-sectional area (-5\% of the nominal value); (iii) evaluating the lack of straightness of the hollow tubes which can be quantified, at first approximation, as an initial imperfection $\mathrm{e}_{0}$ with maximum value of about $1 / 300$ of the member length.

Overall, the preliminary results presented in this work indicate the need of further experiments for the complete assessment of the geometric characterization of Wire-and-Arc Additive Manufactured stainless steel. Further research effort is however needed in order to properly assess the most suitable printing parameters and processes in order to minimize the discrepancies between the digital model and the geometry of the printed outcome. Moreover, specific provisions for the structural design are needed, accounting not only for the mechanical properties, but also for the peculiar geometrical uncertainties of elements produced with this novel manufacturing process.

\section{Acknowledgements}

The support of Dutch company MX3D, which provided the tested materials, is gratefully acknowledged.

\section{Author's contributions}

Conceived the plan: VL, MP, GG, TT; Performed the experiments: VL, MP,VAG; Data analysis: VL, MP, GG, VAG, TT; Wrote the paper: VL, MP, GG, VAG, TT. Authors have no competing financial interests.

\section{References}

1. Carpo, M. (Ed.); The Digital Turn in Architecture 1992-2012; John Wiley \& Sons, Hoboken, 2013.

2. Adriaenssens, S.; Block, P.; Veenendaal, D.; Williams, C. (eds.); Shell Structures for Architecture: Form Finding and Optimization; Routledge, Abingdon, 2014.

3. Khoshnevis, B; Automat Constr, 2004, 13, 5.

4. Attaran, M.; Business Horizons, 2017, 60, 677.

5. Thomas, C.L.; Gaffney, T.M.; Kaza, S.; Lee, C.H.; Proc. Aerospace Applications Conference IEEE, Aspen, CO, 1996, 4 , 219.

6. Song, Y.; Yan, Y.; Zhang, R.; Xu, D.; J. Mater Process Technology, 2002, 120, 237.

7. Giannatsis, J.; Dedoussis,V.; Int. J. Adv. Manuf. Technol., 2009, 40, 116.

8. Buchanan, C.; Gardner, L.; Eng Struct, 2019, 180, 332.
9. Greer, C.; Nycz, A.; Noakes, M.; Richardson, B.; Post, B.; Kurfess, T.; Love, L.; Addit Manuf, 2019, 27, 159.

10. Rodrigues, T. A.; Duarte, V.; Miranda, R. M.; Santos, T. G.; Oliveira, J. P.; Materials, 2019, 12, 1121

11. Wong, K.V.; Hernandez, A.; A review of additive manufacturing, ISRN Mechanical Engineering, 2012.

12. Lewandowski, J.J.; Seifi, M.; Annu. Rev. Mater. Res., 2016, 46, 151.

13. Sames, W.J.; List, F.A.; Pannala, S.; Dehoff, R.R.; Babu, S.S.; Int. Mater. Rev., 2016, 61, 315.

14. Everton, S.K.; Hirsch, M.; Stravroulakis, P.; Leach, R.K.; Clare, A.T.; Mater. Des., 2016, 95, 431.

15. Skiba, T.; Baufeld, B.; Van der Biest, O.; IJIS International, 2009, 49, 1588.

16. Niendorf T.; Leuders, S.; Riemer, A.; Richard, H.A.; Tröster, T.; Schwarze, D.; Metall Mater. Trans. B, 2013, 44, 794.

17. Guan, K.; Wang, Z.; Gao, M.; Li, X.; Zeng, X.; Mater. Des., 2013, 50, 581.

18. Yap, C.Y.; Chua, C.K.; Dong, Z.L.; Liu, Z.H.; Zhang, D.Q.; Loh, L.E.; Sing, S.L.; Appl. Phys. Rev., 2015, 2, 041101.

19. Song, B.; Zhao, X.; Li, S.; Han, C.; Wei, Q.; Wen, S.; Liu, J.; Shi, Y.; Front. Mech. Eng, 2015, 10, 111.

20. Buchanan, C.; Matilainen, V.P.; Salminen, A.; Gardner, L.; J. Constr. Steel Res., 2017, 136, 35.

21. Williams, S.W.; Martina, F.; Addison, A.C.; Ding, J.; Pardal, G.; Colegrove, P.; Mater. Sci. Technol, 2016, 32, 641.

22. Uziel, A.; Weld J., 2016, 4, 42.

23. Haden, C.V.; Zeng, G.; Carter III, F.M.; Ruhl, C.; Krick, B.A.; Harlow, D.G.; Addit Manuf, 2017, 16, 115.

24. Ji, L.; Lu, J.; Liu, C.; Jing, C.; Fan, H.; Ma, S.; MATEC Web of Conferences, 2017, 128, 03006.

25. MX3D company (www.mx3d.com).

26. Joosten, S.K.; Delft University of Technology, 2015, Master thesis.

27. Van Bolderen, G.S.; Delft University of Technology, 2017, Master Thesis.

28. Rios, S.; Colegrove, P.A.; Martina, F.; Williams, S.W.; Addit Manuf, 2018, 21, 651.

29. WAAMMAT (www.waammat.com).

30. Dinovitzer, M.; Chen, X.; Laliberte, J.; Huang, X.; Frei, H.; Addit Manuf, 2019, 26, 138.

31. Xu, X.; Ganguly, S.; Ding, J.; Guo, S.; William, S.W.; Martina, F.; Mater. Charact., 2018, 143, 152.

32. Xu, X.; Ganguly, S.; Ding, J.; Dirisu, P.; Martina, F.; Liu, X.; Williams, S.W.; Mater. Sci. Eng. A, 2019, 747, 111.

33. European Committee for Standardization (CEN), EN 1993-14:2006+A1:2015. Eurocode 3 Design of Steel Structures, Part 1-4: General Rules Supplementary Rules for Stainless Steel, 2015.

34. Rhinoceros Software (www.rhino3d.com).

35. Laghi, V.; Palermo, M.; Gasparini, G.; Girelli, V.A.; Trombetti, T.; J. Constr. Steel Res., 2019 (under review).

36. Laghi, V.; Palermo, M.; Pragliola, M.; Girelli, V.A.; Van Der Velden, G.; Trombetti, T.; Proc. IASS Symposium 2018, 2018 Boston, USA.

37. Artec Spider 3D (www.artec3d.com). 